\title{
"CAUSAS ENDÓGENAS Y EXÓGENAS DEL RENDIMIENTO ACADÉMICO DE LOS ESTUDIANTES DE MATEMÁTICA, COMPUTACIÓN E INFORMÁTICA DE LA FACULTAD DE CIENCIAS DE LA EDUCACIÓN DE LA UNJBG DE TACNA"
}

Responsable: Lic. José Luis Alvarez Quispe

\begin{abstract}
RESUMEN
El rendimiento académico se ha convertido en una problemática, que es preocupación de docentes y autoridades universitarias. El presente trabajo de investigación muestra que no solamente es problema de especialidades profesionales que no son afines a la matemática, sino también en especialidades que tienen que ver con la matemática misma, en este caso con la carrera que forma profesores de matemática. Los estudiantes presentan una heterogénea y deficiente formación académica en al área de matemática, insuficiente capacidad de análisis, abstracción y razonamiento. Carente formación de hábitos y técnicas de estudio. Presentan inmadurez, inseguridad y falta de una orientación vocacional. Desde el punto de vista de los estudiantes los docentes son responsables de su rendimiento deficiente fundamentalmente el carácter, la metodología y la forma de evaluar del docente, así también los estudiantes por la falta de hábitos de estudio y práctica matemática, sobre todo debido a los factores endógenos como estado de salud, habilidades intelectuales y de comunicación, etc.
\end{abstract}

\section{ABSTRACT}

The deficient academic performance in Mathematics has become in a problem for professors and University Authorities, the present investigation work show us, that it is not only a professional career problem which are not related to mathematics, but beside professional careers directly related with the mathematics, in this case with the career that forms mathematics teachers. Students of this career show a heterogeneous and poor academic formation in to mathematics area, deficient ability for analysis, abstraction and reasoning. Students show lack of formation in study habits and Techniques. They are immature, insecure and exist a deficient vocational orientation.

According to students the professors are the responsible of the deficient academic performance, mainly by the professors characters, methodology and evaluation way, beside of bad study habits and lack of mathematics practice.

\section{INTRODUCCIÓN}

El rendimiento académico en la asignatura de matemática en la educación universitaria tiene características de problemática educativa, que en los últimos años presenta diferentes matices, fundamentalmente que la raíz de la problemática nace en niveles anteriores en el sistema educativo nacional. Los docentes y algunos estudiosos de esta realidad coinciden en señalar que los problemas poseen su origen en una serie de factores y enumeran problemas como: enseñanza mecanizada y poco racional de la matemática, docentes con poco afecto a la matemática en los primeros años de la educación primaria, docentes de educación secundaria con poca creatividad en la innovación de recursos académicos para la enseñanza de la matemática, desconocimiento de la realidad inmediata del alumno por parte de los docentes, recargo y falta de conexión de los contenidos curriculares, planes o sumillas de los cursos, inadecuados, metodologías de la enseñanza inadecuadas, planteamiento de problemas y ejercicios al margen de la realidad local y nacional, educación despersonalizada, currículo nointegrado, falta de orientación vocacional, falta de hábitos de estudio, y hasta problemas de tipo social y psicológico en los estudiantes; todos estos problemas frente a un bajo rendimiento de los estudiantes. Por otro lado, la educación secundaria no proporciona una educación adecuada, los exámenes de ingreso a las universidades e instituciones superiores son la prueba de la deficiente formación de los educandos en el nivel secundario, y los elevados porcentajes de estudiantes que recurren a los ciclos preuniversitarios muestran la inseguridad en su preparación en educación secundaria.

Desde el punto de vista técnico-pedagógico, los pedagogos opinan: "Ya en el siglo pasado -dice Dieudonné- se consideraba el paso de la matemática de la escuela secundaria a la Universidad como un salto a un mundo diferente. Con la introducción de las matemáticas modernas, este foso se ha agrandado mucho...". La preparación en las asignaturas, y especialmente en matemática en educación secundaria, no 
satisface a la Universidad, por las apreciaciones de autoridades universitarias respecto a los exámenes de ingreso, como por ejemplo: "En los exámenes de matemática se observa en los postulantes un criterio hábil para aplicar fórmulas y los problemas de este tipo son por lo general bien resueltos; pero cuando se trata de plantear una ecuación o de resolver un problema aritmético en que tenga que aplicar su criterio, entonces fracasan definitivamente". Otro comentario es: "La causa del bajo rendimiento parece ser el tipo de instrucción que reciben en los colegios secundarios, a base de memorización mecánica, que no ejercita las funciones mentales". "Los alumnos han demostrado falta de razonamiento en los diversos planteamientos que las pruebas de exámenes les exigen. Es muy necesario hacer ver que a los alumnos examinados se les debió exigir, en la educación escolar, a razonar en forma lógica y ordenada, dejando a un lado la memorización de los diversos tópicos".

La educación universitaria acoge a estudiantes mayores de 16 años de edad; por lo tanto, se puede decir que se está tratando con adultos y otros en vías a la adultez. Psicológicamente el adulto tiene diferentes características, la Andragogía se plantea como una orientación que se brinda al adulto participante para el desarrollo de su aprendizaje buscando alcanzar su realización positiva y libre en forma horizontal, donde el profesor se convierte en un facilitador del aprendizaje, en oposición a una educación de adultos (vista en forma tradicional) que se fundamenta en el método de la verticalidad donde el profesor es un enseñante, es decir, brinda enseñanza con caracteristicas de educación de menores, sin considerar que el aprendizaje es un proceso personal e individual y al mismo tiempo social.

\section{METODOLOGÍA}

La educación es considerada como una ciencia, una filosofía y un arte. Para un trabajo de investigación experimental tenemos que considerar a la educación como ciencia, al realizar el estudio científico de la conducta de los educandos. El presente trabajo de investigación busca el conocer para hacer, lo que la ubica en el campo de la investigación aplicada. Nos preocupa la aplicación inmediata sobre una realidad circunstancial. Por lo que el método utilizado en la investigación es el método experimental, que lleva a la formulación de una hipótesis que la planteamos de la siguiente manera "Las causas del rendimiento académico de los estudiantes de la especialidad de matemática no sólo están referidas al propio estudiante sino también a los propios docentes y a su entorno".

Se utilizaron instrumentos de recolección de datos empleando las técnicas e instrumentos pertinentes.

\begin{tabular}{|l|l|l|}
\hline Procedimientos & Técnicas & Instrumentos \\
\hline $\begin{array}{l}\text { Personal } \\
\text { Indrecto }\end{array}$ & Observacion & Guia de observación \\
& Entrevista & Guia de entrevista \\
& Encuestas & Cuestionarios \\
\hline
\end{tabular}

\section{FACTORES QUE DETERMINAN EL RENDIMIENTO ACADÉMICO}

Muchos factores influyen en el rendimiento académico, unos que pertenecen o se encuentran en el mismo estudiante (endógenos), y otros que pertenecen o se encuentran en el mundo circundante (exógenos). Estos factores no actúan aisladamente, el rendimiento académico es el resultado de la acción recíproca de lo interno y lo externo.

El rendimiento académico considerado por Isaac Tapia Aréstegui, en su obra "Evaluación", como: "El resultado final de la influencia del proceso educativo, que sintetiza la acción conjunta de sus componentes, orientada por el profesor y lograda por el esfuerzo del alumno, que evidencia la formación integral de éste en sus cambios de conducta de acuerdo a los objetivos previstos". Deja traslucir la presencia de factores diversos que se pueden sintetizar en dos grupos. Uno que engloba los factores que se encuentran fuera de la individualidad del educando, tales como la sociedad, la familia, el educador, etc. $Y$ otro, que agrupa a los factores que se encuentran dentro de la individualidad del educando, como la nutrición, la voluntad, el conocimiento de técnicas de estudio entre las más importantes.

\section{A. Factores Endógenos}

Los factores endógenos que influyen en el rendimiento son:

a. Factores biológicos como, el tipo de sistema nervioso, el estado de salud, el estado nutricional, en general el estado anatómico y fisiológico de todos los órganos, aparatos y sistemas del estudiante.

b. Factores psicológicos como por, la salud mental del estudiante, las características intelectuales, las características afectivas, el lenguaje, etc.

\section{B. Factores Exógenos}

Estos factores son: 
a. Factores sociales como hogar al que pertenece, clase social del estudiante, modo de vida que le es usual, tipo de trabajo que realiza, nivel educacional que posee, etc.

b. Factores pedagógicos como la autoridad educativa, el profesor, el currículo, la metodología de la enseñanza, el sistema de evaluación de los recursos didácticos, el local universitario, el mobiliario, el horario académico, la manera de estudiar, etc.

c. Factores ambientales como la clase de suelo, el tipo de clima, la existencia de parásitos y gérmenes patógenos, la existencia de sustancias tóxicas que contaminan el agua, el suelo y la atmósfera, etc.

\section{EL PROBLEMA DEL RENDIMIENTO ACADÉMICO EN MATEMÁTICA}

El bajo rendimiento académico en matemática de los alumnos en educación secundaria y en educación superior es preocupación permanente de los maestros. Para la mayoría, el contenido disciplinario de la matemática es de naturaleza abstracta, su lenguaje simbólico, y requiere de una curiosa combinación de conceptos, operaciones y discernimiento, para que puede ser útil en la solución de situaciones problemáticas. Como complemento la actividad escolar en matemáticas es compleja y a veces poco comprendida por los propios maestros. Saber matemáticas no garantiza saber enseñar matemáticas, $y$, por otro lado, aunque se tenga una buena formación psicopedagógica, difícilmente puede enseñarse bien un objeto que se desconoce o que se conoce limitadamente. El rendimiento escolar de esta materia, salvo contadas excepciones, es más bajo de lo que se quisiera. En algunos maestros está latente en sus reflexiones como parte de una culpa que no puede ser superada con esfuerzos que se orientan tan sólo con la buena voluntad. Es preciso decir que aunque el rendimiento escolar se manifiesta individualmente, tiene repercusiones de índole social. El paquete cultural que la escuela debe aportar al alumno presupone una mejor preparación para su desempeño en la sociedad, para una existencia más racional y completa y para su propia proyección en el ámbito de la cultura. Si bien los aprendizajes que logren unos alumnos de cualquier especialidad en la Universidad y especialmente en la secundaria va a repercutir en etapas posteriores de manera global, es importante destacar que los resultados que explícita o implícitamente logren van a ser determinantes en la toma de decisiones respecto a sus acciones presente y futuras.

Consideramos que el problema del rendimiento académico en la Universidad, tiene sus raíces en la educación secundaria. Entre los especialistas, preocupa el deterioro que ha tenido la enseñanza de las ciencias, y especialmente la enseñanza de la matemática, aun más allá del colegio secundario, por el reflejo que esto conlleva en la formación científica de las nuevas generaciones y su consecuencia en los aspectos tecnológico y social.

\section{LA MATEMÁTICAEN LAUNIVERSIDAD}

La pedagogía de la matemática tiene que ser revisada profundamente por cada Universidad y cada una de ellas tiene que estudiar lo que está aconteciendo y encontrar soluciones para cada caso. El mundo de hoy requiere más matemática que nunca, pero la gran interrogante que aún no tiene una respuesta es ¿qué hacer en el terreno de la pedagogía de la matemática?, ¿podemos usar la historia como herramienta pedagógica en el aula de clase?. Creo que sí. Y también podemos usar las aplicaciones como una herramienta pedagógica y no dejarlas simplemente como un hecho colateral al final de una capítulo, sino que sea fuente de motivación de los conocimientos matemáticos.

Si sabemos sobre la naturaleza de los diferentes tipos de aprendizaje, si conocemos sobre la naturaleza de la matemática y de su aprendizaje, ¿por qué fracasan los alumnos en matemática, especialmente en los primeros años? Considero que hay varios errores que explican estos deficientes rendimientos, mencionaremos algunos:

a. Enseñanza y Aprendizaje. La mayoría de los docentes de matemática generalmente se preocupan de enseñar y se preocupan muy poco si sus alumnos aprenden. No toman en consideración las condiciones de aprendizaje sugeridas por Gagné para el aprendizaje de la información verbal, habilidades intelectuales, actitudes, etc.

b. Disciplina y Aprendizaje. La mayoría de docentes de matemática se preocupan preferentemente por los contenidos matemáticos, su secuencia, en vez de realizar jerarquías, realizar esfuerzos, generar actitud positiva, etc.

c. Logros de Contenidos y Logros de Aprendizaje. Los docentes se interesan mayormente por cumplir con el desarrollo del programa (silabo). Esto es exigencia del sistema, ya que de acuerdo con las normas 
será supervisado y verificado su cumplimiento. El profesor está alerta a esta exigencia, pero no le preocupa si el porcentaje de desaprobados es alto.

d. Motivación Docente y Motivación del Alumno: El profesor está comprometido con la matemática, le gusta, la disfruta, la ve fácil, etc. Pero generalmente no se genera lo mismo en el alumno. El agente del aprendizaje es el alumno, el debe estar motivado para aprender matemática.

\section{RENDIMIENTO ACADÉMICO EN MATEMÁTICA EN LA ESPECIALIDAD DE MATEMÁTICA, COMPUTACIÓN E INFORMÁTICA DE LA FACULTAD DE CIENCIAS DE LAEDUCACIÓN}

Para el rendimiento académico en la especialidad de matemática, hemos tomado a los estudiantes de letras solamente con un propósito comparativo. Los que nos ha mostrado que los alumnos de la especialidad de matemática tienen un rendimiento ligeramente superior a los alumnos de letras; lo que realmente nos interesa es el rendimiento de los alumnos de la especialidad de matemática, que en este caso hemos todo a los alumnos de la especialidad de matemática, Computación e Informática (MACl) de la Facultad de Ciencias de la Educación (FACE) de la Universidad Nacional Jorge Basadre Grohmann de Tacna; y obtenemos los siguientes resultados:

\begin{tabular}{|c|c|c|}
\hline \multicolumn{3}{|c|}{ RENDIMIENTO ACADÉMICO EN MATEMÁTICA } \\
\hline $\begin{array}{c}\text { CALIFICACIÓN } \\
\text { VIGESIMAL }\end{array}$ & $\begin{array}{c}1^{\circ} \\
\text { ANNO }\end{array}$ & $\begin{array}{c}2^{\circ}, 3^{\circ} \mathbf{Y ~}^{\circ} \\
\text { ANNOS }\end{array}$ \\
\hline $00-05$ & $23 \%$ & $11 \%$ \\
\hline $06-10$ & $27 \%$ & $33 \%$ \\
\hline $11-12$ & $23 \%$ & $37 \%$ \\
\hline $13-15$ & $23 \%$ & $18 \%$ \\
\hline $16-18$ & $3 \%$ & $3 \%$ \\
\hline $19-20$ & $100 \%$ & $100 \%$ \\
\hline
\end{tabular}

El cuadro nos muestra que el porcentaje de desaprobados en los estudiantes ingresantes a la especialidad matemática es de $52 \%$, y los alumnos que tienen un rendimiento regular es de $23 \%$, y los que tienen un rendimiento bueno hacia delante es de $27 \%$.

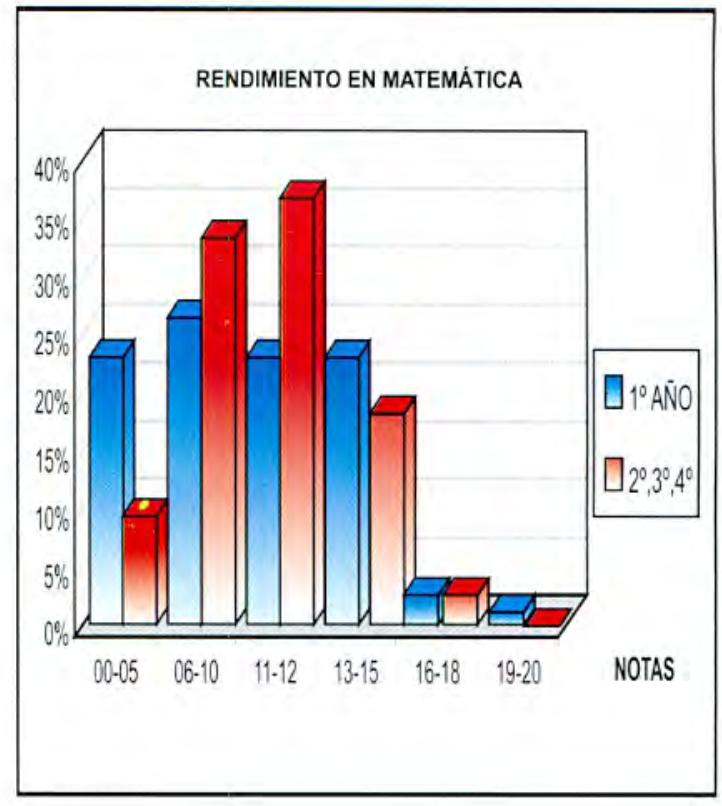

Por otro lado, se observa que el rendimiento de los alumnos de la especialidad de matemática en los años superiores es más elevado, es decir, el porcentaje de desaprobados va disminuyendo respecto al porcentaje de desaprobados del primer año, lo que nos muestra que la madurez hace que el estudiante de los años superiores vaya tomando más conciencia de su papel como estudiante y como futuro profesional.

En los años superiores en la especialidad de matemática se encuentran estudiantes más selectos, debido a la deserción; y, por otro lado, la cantidad de alumnos que se van quedando por deficiencia académica hace que los alumnos de los años superiores sean más selectos.

Estas apreciaciones no justifican que el rendimiento académico de los estudiantes de matemática tengan altos porcentajes de desaprobados y que los aprobados tengan bajas calificaciones, aunque el mayor problema existe en los alumnos del primer año de estudios.

\section{RENDIMIENTO ACADÉMICO DE ACUERDO CON LOS FACTORES}

El rendimiento académico de acuerdo con los factores endógenos y exógenos que influyen en dicho rendimiento se comprueba en resultados que mostramos en un cuadro resumen. En primer lugar, los factores exógenos, los cuales hemos considerado como los pedagógicos, sociales y ambientales; en segundo lugar, los factores endógenos, con sus características biológicas y sicológicas; son los resultados de nuestra investigación de acuerdo con cuestionarios en los que hemos tomado en cuenta características particulares de cada uno de los factores, a través de aptitudes y actitudes de los estudiantes. 


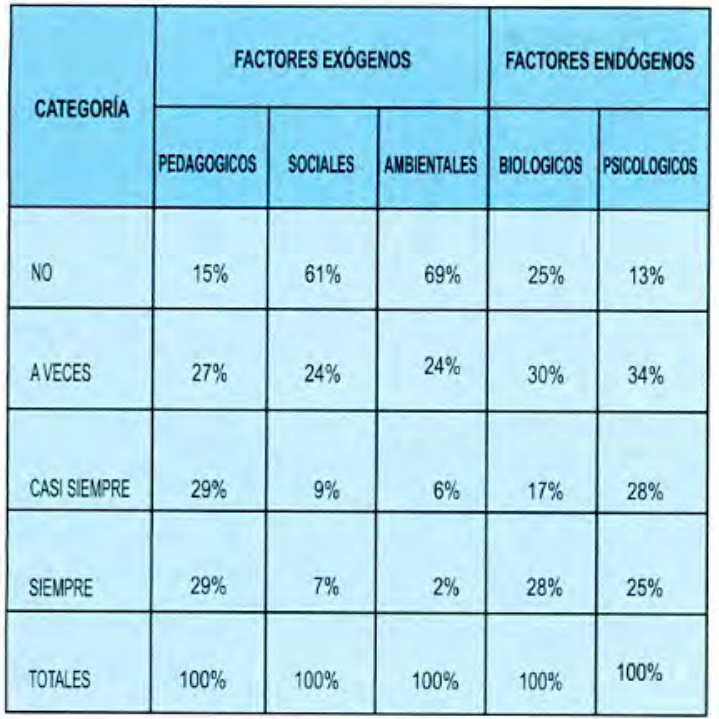

La apreciación de los estudiantes es que de los factores exógenos, la responsabilidad del rendimiento académico es de los docentes, considerando los siguientes aspectos: el carácter, la metodologia, los recursos didácticos, la forma de evaluación de los docentes, es decir, es necesario que se estudie la posibilidad de mejorar estos aspectos. Por otro lado, se observa que los estudiantes reconocen que carecen de hábitos de estudio, tanto en el primer año como en los años superiores. Como se puede observar en los resultados, los estudiantes le dan menos valor a las influencias de los factores exógenos referidos al factor social y ambiental.

Para el estudiante los factores endógenos juegan un papel importante en su rendimiento académico, situaciones como su estado nervioso, su estado de salud, su estado de nutrición; por otro, lado las características intelectuales, las caracteristicas afectivas del alumno y en menor grado las habilidades comunicativas y la salud mental del estudiante. Estas caracteristicas generalmente no son tomadas en cuenta por los docentes, lo que deben ser una preocupación no solamente de los docentes de la especialidad de matemáticas, sino también de las otras especialidades de la Facultad de Educación y de la Universidad en general.

\section{CONCLUSIONES}

1. Se observa una influencia negativa de las academias de preparación, puesto que tienden a automatizar al estudiante proporcionándole solamente entrenamiento para el examen de ingreso, sin proporcionarle la capacidad de análisis y razonamiento. Además de la insuficiente preparación debido al corto periodo de dichas academias. La carencia de hábitos y técnicas de estudio. La escasa capacidad de abstracción, generalización y análisis. La inmadurez, inseguridad y falta de una orientación vocacional.

2. Sobre los contenidos básicos curriculares, se puede decir que existe una heterogeneidad en los programas de matemática de educación secundaria, así como una discontinuidad con los de nivel universitario. La heterogeneidad de los contenidos programáticos de la asignatura de matemática ofrecido a los ingresantes a la Universidad no brinda una cultura matemática básica al estudiante.

3. Sobre la metodología, el docente universitario en el área de matemática no aplica su formación metodológica, que permita optimizar el proceso enseñanza-aprendizaje, lo cual se refleja en la falta de interrelación profesor-alumno, la ausencia de motivación en el proceso enseñanza aprendizaje, la priorización de la enseñanza antes que el aprendizaje del estudiante, la carencia de textos adecuados de matemática para el primer año universitario, la falta de equipos y material didáctico para el proceso de enseñanza (retroproyectores, microcomputadoras, cañón de proyección, etc.)

4. Sobre la evaluación, podemos decir que, en muchos casos, los docentes desconocen los criterios educacionales indispensables para la elaboración adecuada de las prácticas y exámenes, muchas veces se incide en preguntas rebuscadas y sutilmente planteadas. Las pruebas elaboradas tienden a evaluar cuantitativamente y no cualitativamente el aprendizaje del estudiante.

5. Dentro de las causas del bajo rendimiento académico de carácter exógeno, son las aplicaciones de las cualidades del docentes, su preocupación por el rendimiento de los estudiantes debe ser de mayor preocupación por parte del docente. Es imprescindible que se modifique la metodología, las formas de evaluación, desterrar algunos mitos sobre las asignaturas de matemática, respecto a la cantidad de desaprobados, reformular las programaciones, sobre todo en las áreas de letras.

6. Los estudiantes consideran que los factores endógenos tienen mucho que ver con el bajo rendimiento académico en las áreas de matemática, lo que demuestra que el estudiante carece de un actitud positiva hacia el estudio, la evaluación y el rendimiento de los cursos de matemática.

\section{RECOMENDACIONES}

- A través de convenios entre el Ministerio de Educación y la Universidad se realice un estudio integral sobre la enseñanza de la matemática a nivel primario y secundario a fin de reformular los contenidos programáticos recuperando su carácter formativo cultura y aplicativo.

- Solicitar a las autoridades correspondientes del Ministerio de Educación una mejor fiscalización en el cumplimiento de los programas curriculares en 
el nivel primario y secundario.

- Brindar al recién ingresante a la Universidad, a través de un ciclo básico, una formación cientifica y humanística integral que le permita continuar apropiadamente sus estudios profesionales en la especialidad elegida. $Y$ sugirar que sea para toda la Universidad.

- Elaborar contenidos programáticos de matemática básica universidataria con participación de todos los profesores de dicha asignatura en la Facultad de Ciencias de la Educación y en coordinación con las diversidad facultades si es que el proyecto fuese a nivel de la Universidad.

- Promover el uso de equipos y medios audiovisuales en el proceso de enseñanza aprendizaje, crear una oficina de material educativo para matemática y todas las especialidades en la Facultad.

- Capacitar y actualizar a los profesores de todos los niveles educativos tanto en los conocimientos básicos como en la didáctica de la matemática.

- Elaborar textos guías a cargo de los profesores de la asignatura, de acuerdo a las necesidades de cada especialidad, contando con el apoyo editorial correspondiente.

- La evaluación del aprendizaje de los estudiantes debe realizarse teniendo en cuenta Los objetivos específicos planteados en el sílabo de la asignatura.

- Los aspectos teóricos y prácticos desarrollados en el curso.

- La graduación de las preguntas de acuerdo a niveles de dificultad diversa y al tiempo que dure la evaluación.

- El empleo de instrucciones claras y precisas.

- Sugerir las evaluaciones únicas anuales, semestrales o bimestrales (según las posibilidades) para la educación secundaria, en coordinación las Facultades de Educación de la
Universidad. Con la finalidad de uniformizar los rendimientos y supervisar el proceso de enseñanza aprendizaje y aplicar los correctivos enforma oportuna.

\section{BIBLIOGRAFÍA}

Alves de Matos, Luiz , (1970). "Compendio de Didáctica General'. Editorial Kapeluz, Buenos Aires.

Bent Kronenberg, (1970). "Fundamentos de la Educación Secundaria" Edit. HISPANO AMERICA, México.

Beard, Ruth, (1974). Pedagogia y Didáctica de la Enseñanza Universitaria, Barcelona España, Editorial OIKOS TAU.

Castel Nuevo, Emm, (1987). "Didáctica de la Matemática", Editorial Trillas, México.

Cruz Valverde, Aurelio, (1970). "El Sistema de Planeación y el Diagnóstico de la Educación Superior",Editorial Trillas, México.

Diaz Bordonave, Juan. MARTINS PEREIRA, Adair (S/A). Estrategias de la Enseñanza Aprendizaje, Orientaciones Didácticas para la Docencia Univesitaria, San José Csta Rica, Editorial Instituto Interamericano de Cooperación para la Agricultura.

Dottrens, Rober, (1970). "Como Mejorar los Programas Escolares", Editorial Kapeluz, Buenos Aires,

Harold, Benjamín, (1970). "La Educación Superior en las Repúblicas Americanas", España. Ediciones del Castillo.

Pansza G., Margarita y Colaboradores, (1993). Fundamentación de la Didáctica, Tomo 1, 6 ta. Edición, México, Editorial Gernika.

Pansza G., Margarita Pérez J. Esther C. Morano, Porfirio (1993). Operatividad de la Didactica, Tomo li. 5ta. Edición, México. Editorial Gernika.

Rodriguez D.,J. Luis (1986). "Didáctica General”, "Editorial Cincel, Madrid-España”. 